\title{
depósito elevado, en Belfast
}

FREDERICK GIBBERD CBE, arquitecto

FELIX J. SAMUELY \& PARTNERS

\section{simopsis}

Entre las distintas construcciones que constituyen el moderno hospital Ulster de Dundonald, en Belfast (Irlanda del Norte), destaca, particularmente, el depósito elevado para suministro y reserva de agua.

Desde el punto de vista arquitectónico, su forma es de plástica agrada ble y de linea suave y graciosa, puescopa de gran tamañ muy utilizada. Su estructura, de hormi ón armado, es sencilla $y$ ha permitido aprovechar el núcleo central o soporte hueco, para chimenea de la sala de calderas. La altura total del depósito es de $32,50 \mathrm{~m}$ y su capacidad de $450 \mathrm{~m}^{3}$. EI depósito propiamente dicho tiene $15,5 \mathrm{~m}$ de altura y $10 \mathrm{~m}$ de diámetro máximo. El espesor de las paredes varia de 0,32 a $0,15 \mathrm{~m}$. Las anillas de traccion de las armaduras estan formadas con barras de $19 \mathrm{~mm}$ de diámetro, mientras que las armaduras radiales son barras de $9 \mathrm{~mm}$ de diámetro.

El núcleo central o chimenea lleva una pared de $0,15 \mathrm{~m}$ de espesor, $y$ ras verticales están constituidas barras de $25 \mathrm{~mm}$ de diámetro. riormente, este núcleo o soporte presenta ocho nervios que se proyectan al exterior y tienen una sección de 0,20 de anchura por $0,30 \mathrm{~m}$ de profundidad. Toda la estructura se apoya sobre una losa octogonal de $9 \mathrm{~m}$ entre vértices opuestos y de $2 \mathrm{~m}$ de espesor. La tension maxima prevista para el hormigon, trabajando a tracción y en las condiciones más desfavorables, es de $12 \mathrm{~kg} / \mathrm{cm}^{2}$.

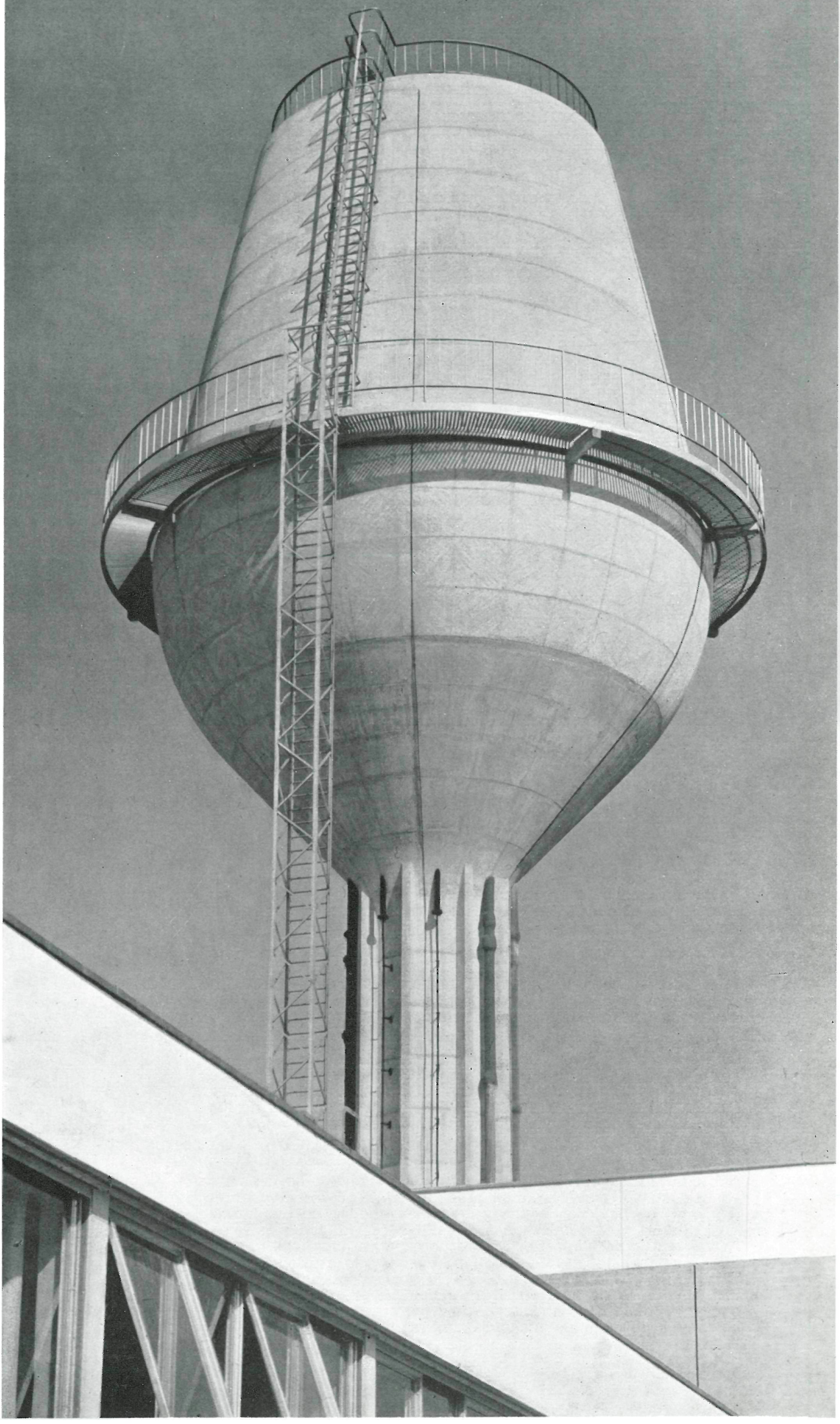




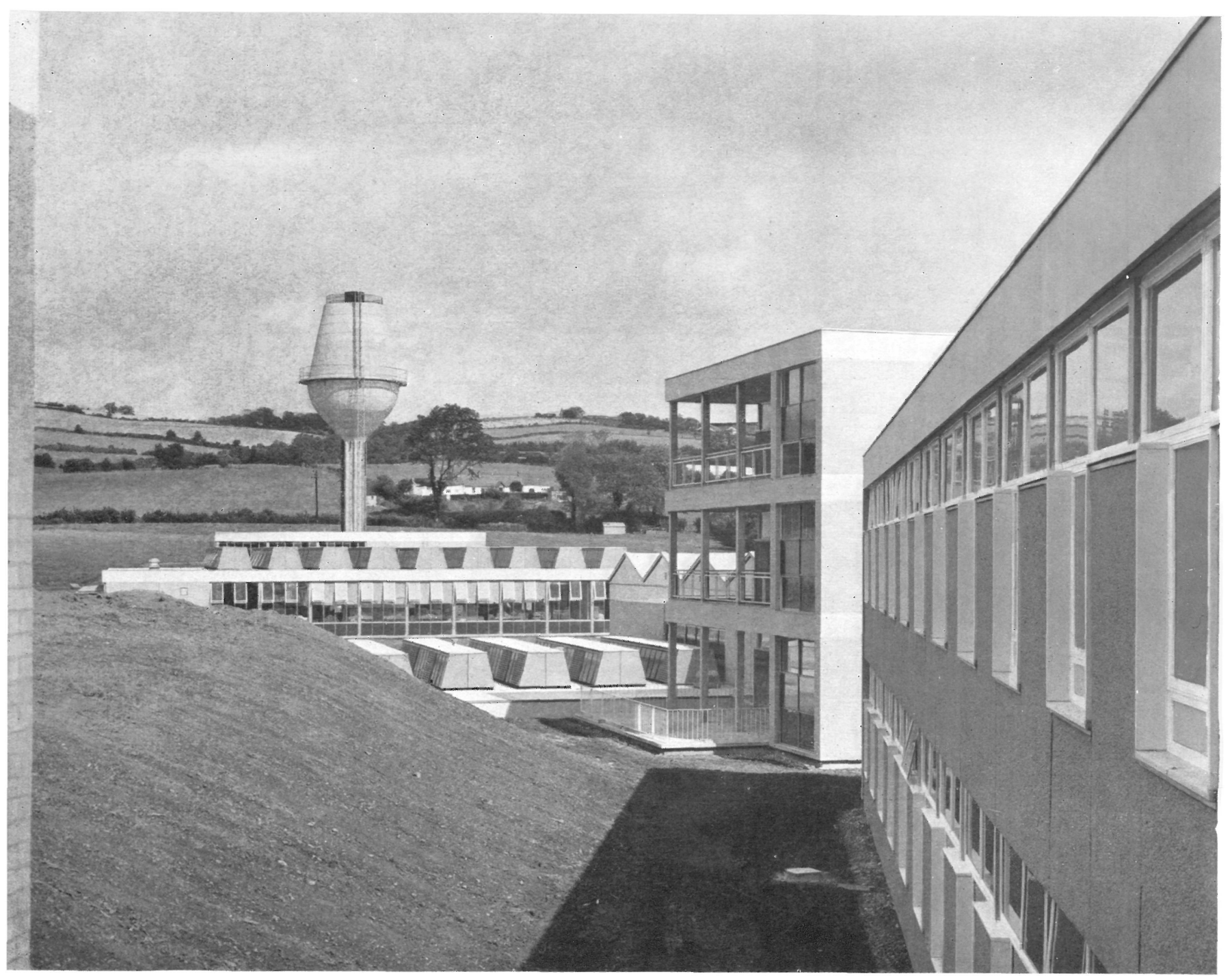

Vista general del conjunto del hospital.

Cuerpo central y entramado tubular auxiliar.

Recientemente se ha terminado el conjunto de obras que constituyen el nuevo Hospital Ulster, de Dundonald, en Belfast (Irlanda del Norte).

En este artículo sólo trataremos de una de estas obras: del depósito elevado para agua y reserva del hospital. En su estudio y proyecto se han integrado, en un solo cuerpo, el deposito la forma el hueco interior del soporte cilíndrico de aquél.

La construcción tiene $32,50 \mathrm{~m}$ de altura, y su capacidad es de 450 metros cúbicos. El aspecto externo del depósito es gracioso, ya que se parece a una copa de gran tamaño o, si se quiere, a un tulipán. El cuerpo central o soporte sobresale por encima del depósito, para dar salida libre a los humos de los
hogares de la sala de calderas.

Toda la estructura es de hormigón armado, salvo la parte interior del cuerpo central cilindrico, hueco, que se ha revestido con ladrillo refractario. La superficie exterior del depósito es lisa, suave y corresponde a la obtenida al desencofrar el depósito, haciendose en ella patente las huellas de las juntas horizontales y verticales de los encofrados utilizados para el hormigonado del depósito. El cuerpo central o soporte, de sección circular, de $2,10 \mathrm{~m}$ de diámetro, está provisto de ocho nervios de $0,20 \mathrm{~m}$ de anchura y $0,30 \mathrm{~m}$ de profundidad según una sección plana horizontal. La superficie exterior de la gran copa y la del fuste provisto de nervios crea un contraste gracioso y acentuado de luces y sombras.

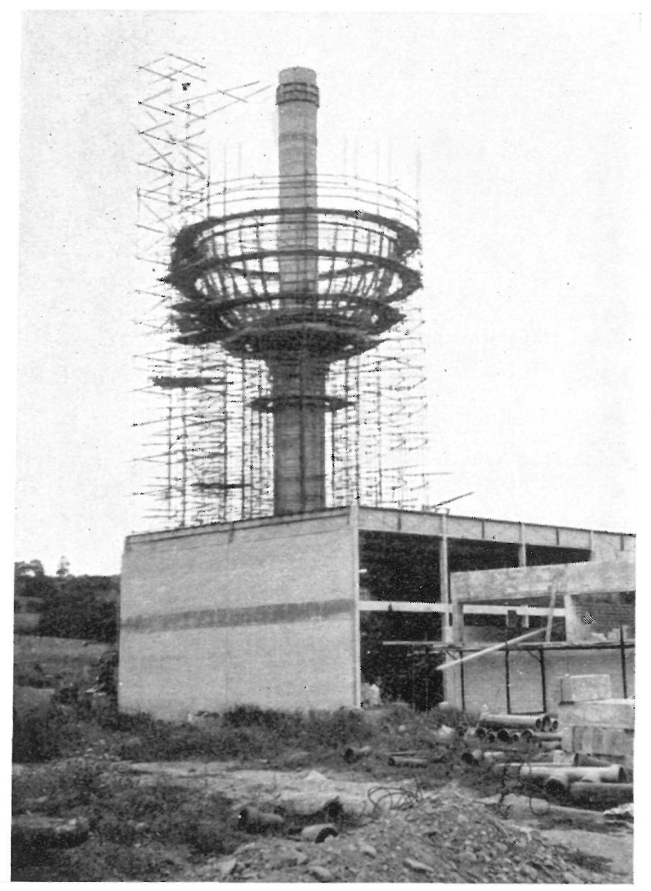




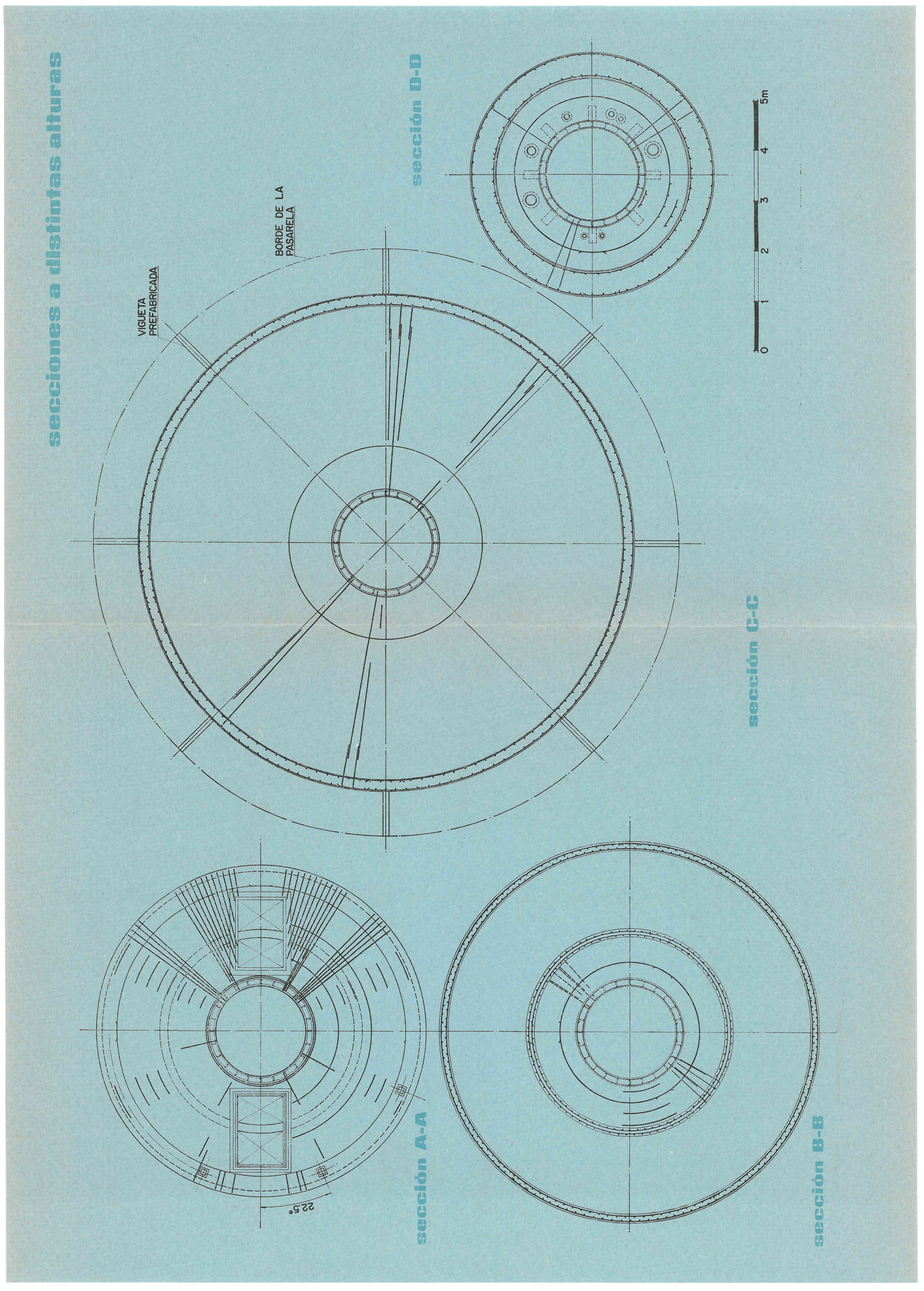




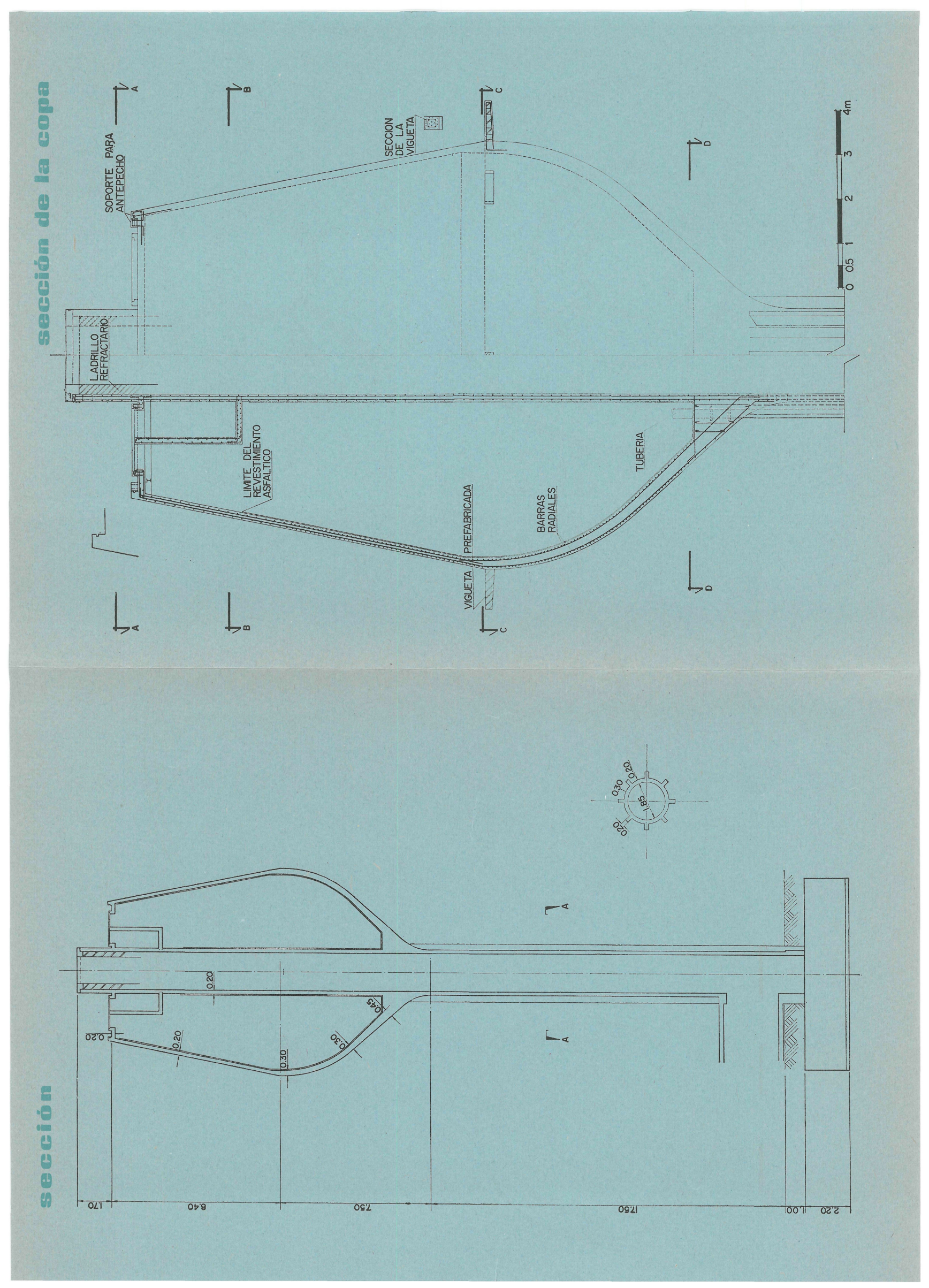



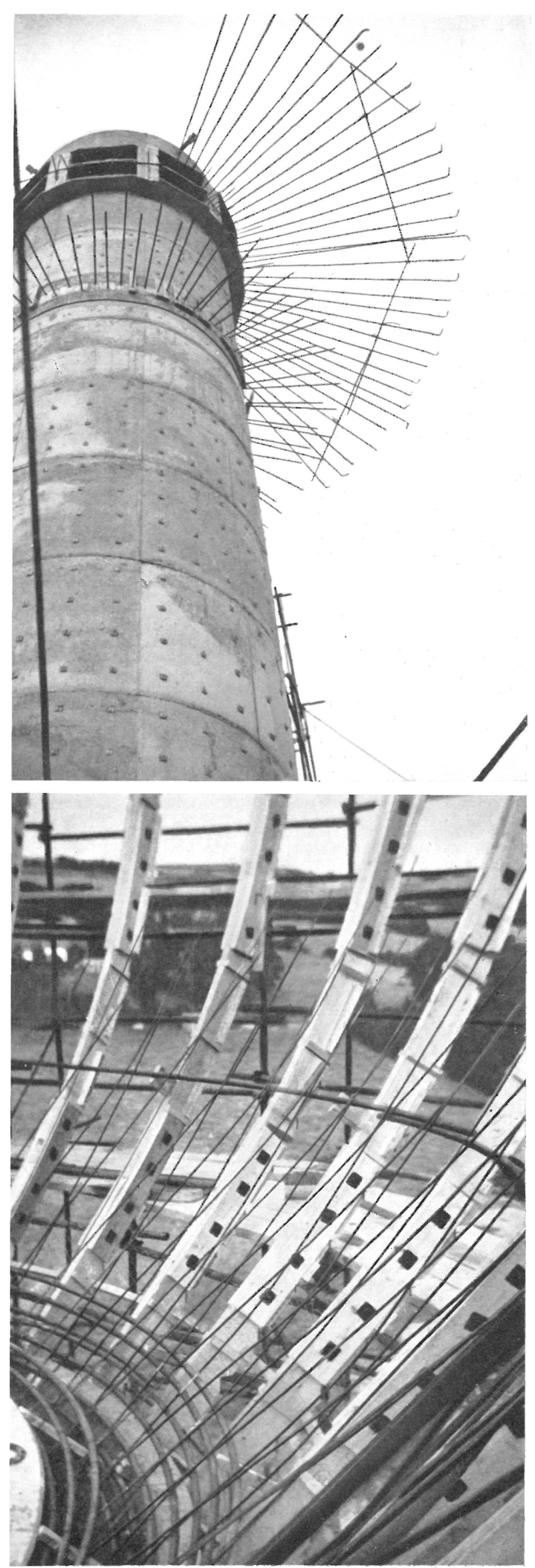

Armaduras para la cubierta.

Anillos de traccióa.

En la zona que corresponde al diámetro máximo del depósito se ha montado una pasarela, de aluminio, soportada por viguetas, de hormigón en voladizo, que emergen de la pared del depósito, dispuestas así durante la construcción de dicha pared. El piso de esta pasarela es un reticulado de barras circulares y radiales, en cuya parte exterior se ha levantado un antepecho o barandilla El acceso a esta pasarela, así como a la cubierta del depósito, se realiza por medio de escaleras también de aluminio. El cuerpo del propio depósito tiene $15,5 \mathrm{~m}$ de altura y un diámetro máximo exterior de $10 \mathrm{~m}$. El espesor de la pared varía de 0,32 a $0,15 \mathrm{~m}$, y se ha reforzado con armaduras de barras, de $19 \mathrm{~mm}$ de diámetro, que forman los anillos de tracción, y barras radiales, de $9 \mathrm{~mm}$ de diámetro y de dirección normal a la de dichos anillos.

La estructura se apoya sobre un cimiento, octogonal, de $9 \mathrm{~m}$ de diámetro y de $2 \mathrm{~m}$ de espesor. La tensión máxima prevista para el hormigón trabajando a tracción es de $12 \mathrm{~kg} / \mathrm{cm}^{2}$ y en las condiciones más desfavorables. Con objeto de conseguir una impermeabilización perfecta, el interior del depósito se ha revestido con una capa de asfalto. Los conductos de humo llegan al cuerpo hueco central a través de una galería horizontal construida a nivel del suelo.

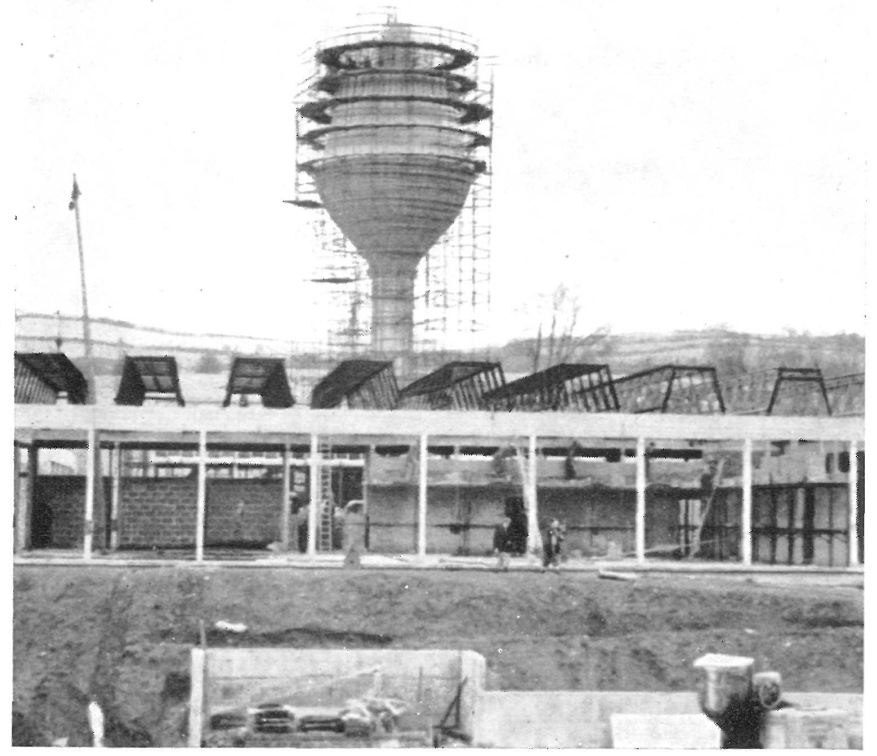

Fase constructiva final.

La estructura, no obstante su aparente sencillez, ha presentado problemas, ya que debían conjugarse dos tipos diferentes de estructura en un cuerpo: el depósito propiamente dicho y la chimenea Esto ha exigido un cuidadoso estudio hasta dar con la solución racional que se perseguía, la cual, teniendo en cuenta la cran masa de agua que el depósito soporta con un centro de gravedad situado a un nivel elevado respecto al suelo, cas de gracias a las características propias del hormigón armado, material que se presta a realizaciones en un tiempo insospechadas.

La parte arquitectónica de esta obra fue concebida por el arquitecto Frederick Gibberd CBE, el constructor fue stewart \& Partners Ltd. La Cement and Concrete Association ha facilitado la información necesaria para la descripción de esta importante obra. 


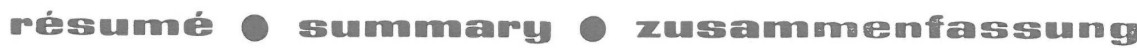

\section{Elhâtrear d"ecur ì Belfast}

Felix J. Samuely \& Partners, ingénieur.

Parmi les différents édifices dont est composé l'hôpital moderne Ulster de Dundonald, à Belfast (Irlande du Nord), on remarque particulièrement le château d'eau.

Du point de vue arehitectural, sa forme, agréable et originale, reproduit la silhouette d'un verre à pied, d'un gracieux effet.

Sa structure, en béton armé, est simple et a permis d'utiliser le noyau central ou support, creux, comme cheminée de la salle de chaudières. La hauteur totale du château d'eau est de $32,50 \mathrm{~m}$ et sa capacité de $450 \mathrm{~m}^{3}$. Le réservoir, proprement dit, a $15,5 \mathrm{~m}$ de haut et $10 \mathrm{~m}$ de diamètre maximum. L'épaisseur de ses murs varie entre 0,32 et $0,15 \mathrm{~m}$. Les anneaux de traction des armatures sont formés par des barres de $19 \mathrm{~mm}$ de diamètre, tandis que les armatures radiales sont des barres de $9 \mathrm{~mm}$ de diamètre.

Le mur du noyau central ou cheminée a $0,15 \mathrm{~m}$ d'epaisseur et est de section circulaire. Les armatures verticales sont formées par des barres de $25 \mathrm{~mm}$ de diamètre. A l'extérieur, ce noyau ou support présente huit nervures qui se projettent à l'extérieur et dont la section a $0,20 \mathrm{~m}$ de large sur $0,30 \mathrm{~m}$ de profondeur.

Toute la structure s'appuie sur une dalle, octogonale, de $9 \mathrm{~m}$ entre sommets opposés et de $2 \mathrm{~m}$ d'épaisseur.

La contrainte maximale prévue pour le béton soumis à la traction et dans les conditions les plus défavorables est de $12 \mathrm{~kg} / \mathrm{cm}^{2}$.

\section{Elevated Tank in Belfast}

Felix J. Samuely \& Partners, engineers.

An outstanding feature of the buildings which constitute the modern Ulster Hospital, at Dundonald, Belfast, is the elevated tant which supplies the hospital with water.

The shape of this tank is pleasant and it provides a charming and smooth outline against the sky: it is reminiscent of a large sized drinking cup.

The structure, made of reinforced concrete, is of straighforward design. The central support is used as the chimney stack for the boiler room. The water tank has an overall height of $32.5 \mathrm{~m}$ and will hold 450 cubic metres of water. The tank for the boiler room. The water tank has an overall height of $32.5 \mathrm{~m}$ and will hold 450 cubic metres of water. The tank
itself is $15.5 \mathrm{~m}$ high and has a maximum diameter of $10 \mathrm{~m}$. The walls vary in thickness between 0.32 and $0.15 \mathrm{~m}$. The hoop reinforcement is provided by $19 \mathrm{~mm}$ bars, whilst the radial reinforcement consists of $9 \mathrm{~mm}$ wires.

The central column, or stack, has a wall thickness of $0.15 \mathrm{~m}$, and is of circular cross section. Vertical $25 \mathrm{~mm}$ rods provide the metal reinforcement. Externally, this central column has eight ribs, $20 \mathrm{~cm}$ wide and $30 \mathrm{~cm}$ deep.

The whole structure rests on an octogonal slab, with a maximum horizontal dimension of $9 \mathrm{~m}$ and is $2 \mathrm{~m}$ thick.

The maximum tensile strength allowed for the concrete, when working in the most unsatisfactory conditions, is $12 \mathrm{~kg} / \mathrm{cm}^{2}$.

\section{Emhöhter Wasserbehäilter in Belfust}

Felix J. Samuely und Partners, Ingenieure.

Unter den verschiedenen Bauten des modernen Krankenhauses Ulster de Dundonald in Belfast (Nordirrland) hebt sich besonders der Wasserbehälter hervor.

Vom architektonischen Standpunkt aus gesehen ist er angenehm anzuschauen. Seine Silhouette ist eine übergrosse stilisierte Tasse mit weicher, harmonischer Linienführung.

Seine Stahlbetonstruktur ist einfach und hat es erlaubt, die im Mittelpunkt befindliche Säule hohl als Schornstein für den Heizungsraum auszunützen. Die Gesamthöhe des Behälters beläuft sich auf $32,50 \mathrm{~m}$ und sein Fassungsvermögen beträgt $450 \mathrm{~m}^{3}$. Der eigentliche Behälterraum hat eine Höhe von $15,5 \mathrm{~m}$ und einen maximalen Durchmesser von $10 \mathrm{~m}$. Die Dicke der Wände schwankt zwischen 0,32 und $0,15 \mathrm{~m}$. Die Zugbewehrung besteht aus Stäben mit $19 \mathrm{~mm}$ Durchmesser, während die radiale Bewehrung sich aus Stäben mit $9 \mathrm{~mm}$ Durchmesser zusammensetzt.

Die mittlere als Schornstein ausgebildete Säule hat eine Wand von 0,15 m Dicke und ist im Querschnitt rund. Die senkrechte Bewehrung besteht aus $25 \mathrm{~mm}$ dicken Stäben. Von aussen her weist die Säule 8 Rippen auf, die einen Querschnitt von $0,30 \mathrm{~m}$ Tiefe und $0,20 \mathrm{~m}$ Breite haben.

Die ganze Struktur stützt sich auf eine $2 \mathrm{~m}$ dicke achteckige Platte mit $9 \mathrm{~m}$ Abstand zwischen den gegenüberliegenden Scheiteln.

Die maximale Spannung, die der Beton bei ungünstigsten Bedingungen aushalten kann, beträgt $12 \mathrm{~kg} / \mathrm{cm}^{2}$. 